\title{
HUMAN/VECTOR RELATIONSHIPS DURING HUMAN AFRICAN TRYPANOSOMIASIS: INITIAL SCREENING OF IMMUNOGENIC SALIVARY PROTEINS OF GLOSSINA SPECIES
}

\author{
ANNE POINSIGNON,* SYLVIE CORNELIE, FRANCK REMOUE, PASCAL GRÉBAUT, DAVID COURTIN, \\ ANDRE GARCIA, AND FRANCOIS SIMONDON \\ Epidémiologie et Prévention, et Interactions Hôtes-Vecteurs-Parasites dans les Trypanosomoses Unité de Recherche, Institut de \\ Recherche pour le Développement, Montpellier, France; Epidémiologie et Prévention Unité de Recherche, Institut de Recherche pour \\ le Développement, Dakar, Senegal; Santé de la Mère et de L'Enfant en Milieu Tropical Unité de Recherche, Institut de Recherche \\ pour le Développement, Paris, France; Santé de la Mère et de L'Enfant en Milieu Tropical Unité de Recherche, Institut de Recherche \\ pour le Développement, Cotonou, Benin
}

\begin{abstract}
The morbidity and mortality of vector-borne diseases is closely linked to exposure of the human host to vectors. Qualitative and quantitative evaluation of individual exposure to arthropod bites by investigation of the specific immune response to vector saliva would make it possible to monitor individuals at risk of vectorial transmission of pathogens. The objective of this study was to evaluate and compare the antibody (IgG) response to saliva from uninfected Glossina species, vectors, or non-vectors of Trypanosoma brucei gambiense by detecting immunogenic proteins in humans residing in an area endemic for human African trypanosomiasis in the Democratic Republic of Congo. Our results suggest that the immunogenic profiles observed seemed specific to the Glossina species (vector or non-vector species) and to the infectious status of exposed individuals (infected or not infected). This preliminary work tends to support the feasibility of development of an epidemiologic tool based on this antibody response to salivary proteins.
\end{abstract}

\section{INTRODUCTION}

The chronic form of sleeping sickness (human African trypanosomiasis [HAT]) caused by Trypanosoma brucei gambiense is found in western and central Africa and is transmitted to humans by bites of the Glossina vector (tsetse fly). Among vector Glossina species, Glossina palpalis, G. tachinoides, and G. fuscipes are able to transmit $T$. b. gambiense. This slowly progressing fatal disease often remains undiagnosed because of lack of specific symptoms observed during its initial stage, and the infection is readily confused with other febrile illnesses, especially malaria. ${ }^{1}$ The World Health Organization (WHO) has recommended systematic screening of populations by serologic survey to diagnose infected individuals and to control HAT transmission. ${ }^{2}$ In the mid1980s, the incidence of HAT increased to alarming levels comparable to those of the early 20th century, particularly in the Democratic Republic of Congo, and it now represents a major public health problem. ${ }^{3}$ This re-emergence can be partly explained by laxity in control and research efforts. In view of this alarming situation, intensification of active case detection and treatment was initiated by the national control programs of disease-endemic countries and results seem promising; WHO recently reported a decrease in the number of new reported cases. $^{4}$

The morbidity and mortality of sleeping sickness is closely linked to transmission of the pathogen by the vector, and is therefore associated with exposure of the human host to infected Glossina bites. However, the relationship between the presence of vectors and the prevalence of the disease is complex, and other parameters must be considered when evaluating risk of infection. ${ }^{5,6}$ This complexity underlines the importance of developing new tools to identify populations ex-

* Address correspondence to Anne Poinsignon, Epidémiologie and Prévention, Unité de Recherche 024, Institut de Recherche pour le Développement, 911 Avenue Agropolis, BP 64501, 34394 Montpellier CEDEX 5, France. E-mail: anne.poinsignon@mpl.ird.fr posed to a high risk of transmission and which could enable optimization of epidemiologic surveillance and identification of individuals who may benefit from monitoring. Currently, exposure of populations to the Glossina vector is evaluated by entomologic methods (e.g., capture by traps), but such methods cannot evaluate heterogeneous individual exposure. ${ }^{7}$

During blood feeding, insects inject salivary proteins (sialome), primarily to counteract the host hemostasis response induced by the bite. The pharmacologic properties of these proteins, such as platelet aggregation inhibitors, vasodilators, and inhibitors of blood coagulation, are necessary for blood feeding. ${ }^{8}$ Some of these salivary proteins also have immunogenic characteristics (immuno-sialome) that enable initiation of a specific immune response. ${ }^{9}$ Based on these immune properties, studies have shown the potential of markers of exposure to vector-borne diseases by evaluation of a specific antibody response to salivary proteins in individuals exposed to the arthropod vector. The first example of an epidemiologic indicator was reported in the United States in individuals infected with Borrelia burgdorferi, the causative agent of Lyme disease. ${ }^{10}$ The level of IgG antibody specific for Ixodes damini tick saliva was higher in individuals at high risk of developing Lyme disease. ${ }^{11}$ Moreover, specific IgG1 and IgG4 isotypes of antibody to Triatoma salivary antigens have been detected in individuals exposed to Chagas disease and the level of specific IgG4 of Aedes aegypti saliva might be a marker of intense exposure to Aedes bites. ${ }^{12-15}$ It has been also suggested that immune response to sand fly recombinant salivary gland protein may be a good marker of vector exposure. ${ }^{16}$ Recently, it has been shown that children from a malaria-endemic area in Senegal developed a specific IgG response to Anopheles gambiae saliva that was positively associated with the degree of exposure to vector bites evaluated by classic entomologic studies. ${ }^{17}$ In addition, high levels of IgG antibody to saliva appeared to be a predictive indicator of malaria morbidity.

Few studies have explored the immunogenic properties of Glossina saliva in sleeping sickness. Immediate or delayed 
hypersensitivity responses have been observed in humans and rabbits exposed to tsetse fly bites, suggesting immunogenic properties of Glossina saliva. ${ }^{18,19}$ Another study attempted to identify an epidemiologic indicator of human contact with Glossina by detection of specific antibody of Glossina salivary glands in human sera, but the immunologic techniques used (immunoelectrodiffusion) failed to detect an immunologic response. $^{20}$

The present study represents a new attempt to evaluate the immune response to tsetse fly bites in human populations in an area endemic for sleeping sickness. The objective of the study was to detect immunogenic salivary proteins of Glossina (vector and non-vector species) and to compare the profiles of these immunogenic proteins, taking into account the infectious status of individuals living in an area endemic for HAT.

\section{MATERIALS AND METHODS}

Studied population. The study was conducted using sera from individuals living in the HAT-endemic area of Bandundu in the Democratic Republic of Congo. ${ }^{21}$ The status of 74 individuals included in the study was defined using serologic (card agglutination trypanosoma tests for T. b.gambiense and trypanolysis test), parasitologic, and molecular (polymerase chain reaction) investigations. ${ }^{22,23}$ Stage diagnosis was carried out for all sleeping sickness cases $(\mathrm{n}=19)$, by counting white blood cells present in the cerebrospinal fluid (CSF). These results led to defining three groups of individuals: 1) the ENI group, which included exposed but non-infected individuals ( $\mathrm{n}=55$ ) who had no positive test results; 2 ) the P1 group, which included exposed and infected patients in the first stage of the disease $(n=7)$ who had positive test results and $<5$ white blood cells/ $\mu \mathrm{L}$ in CSF; and 3) the P2 group, which included exposed and infected patients in the second stage of the disease $(\mathrm{n}=12)$ who had positive test results and $\geq 5$ white blood cells/ $\mu$ in the CSF. A negative control (CHU) group included individuals $(\mathrm{n}=16)$ who were never exposed to Glossina spp. bites; it included personnel from Lapeyronie Hospital (Montpellier, France). The study adhered to the ethical principles defined by the Helsinki Declaration, and was reviewed and approved by local traditional authorities and by the local Ethical Committee of the Democratic Republic of Congo (Public Health Ministry, 2001). All individuals enrolled in this study signed an informed consent form.

Saliva collection. Saliva samples from uninfected male and female Glossina bred in an insectarium (Unité de Recherche 177, Institut de Recherche pour le Développement) were collected. The tsetse flies were enclosed in a tube by a mosquito net and the tube was placed above a drop of salivation buffer (10 mM HEPES, $150 \mathrm{mM} \mathrm{NaCl}$, and $5 \mathrm{mM}$ EDTA, pH 7,2) on warm slides $\left(37^{\circ} \mathrm{C}\right) .^{24}$ After 10 minutes of salivation, the saliva solution was collected, pooled, and stored at $-20^{\circ} \mathrm{C}$ before use. The saliva of four tsetse flies species was collected: three vector species of T. b. gambiense: (G. fuscipes fuscipes, $G$. tachinoides, and G. palpalis gambiensis) and one nonvector species of T. b. gambiense (G. morsitans morsitans).

Sodium dodecyl sulfate-polyacrylamide gel electrophoresis (SDS-PAGE). Prior to electrophoresis, salivary proteins were precipitated. A volume of salivary samples was diluted with nine volumes of ice-cold $\left(-20^{\circ} \mathrm{C}\right) 80 \%$ ethanol. The mixtures were incubated for one hour at $-20^{\circ} \mathrm{C}$ and centrifuged at
$13,000 \mathrm{rpm}$ at $4^{\circ} \mathrm{C}$ for 30 minutes. The supernatants were removed and $1 \mathrm{~mL}$ of ice-cold $\left(-20^{\circ} \mathrm{C}\right) 70 \%$ ethanol was added. Samples were centrifuged as above for 20 minutes. The supernatants were removed and the pellets were airdried. For one-dimensional gel electrophoresis, pellets were suspended in a mixture of $5 \times$ Laemmli:salivation buffer (1:4). Based on the evaluation of the protein concentration by bicinchoninic acid (BCA) test (BCA Protein Assay Kit, Pierce, Rockford, IL), equal quantities of Glossina salivary proteins $(7 \mu \mathrm{g})$ were deposited in each well for electrophoresis. Salivary proteins were separated by one-dimensional electrophoresis on a $4-15 \%$ gradient acrylamide gel in Tris-glycine buffer with SDS (Ready gels Tris-HCl; Bio-Rad Laboratories, Hercules, CA) according to the manufacturer's instructions. Separated proteins were observed by staining with silver nitrate solution (Sigma-Aldrich, St. Louis, MO) based on a modified method of Shevchenko and others. ${ }^{25}$ Gels were fixed twice for 30 minutes in $30 \%$ ethanol and $5 \%$ acetic acid and then washed four times in MilliQ water. Gels were sensitized for one minute in $0.02 \%$ sodium thiosulfate, washed twice (one minute/wash) with Milli/Q water, incubated in $0.1 \%$ silver nitrate containing $0.06 \%$ formaldehyde for 45 minutes, and washed with MilliQ water. Proteins were then visualized in developing solution $(0.05 \%$ sodium carbonate, $0.06 \%$ formaldehyde; Sigma-Aldrich) until a desired level of staining was achieved, after which development was stopped with $5 \%$ acetic acid.

Immunoblotting. The proteins separated by onedimensional electrophoresis were transferred onto a polyvinylidene difluoride membrane (Bio-Rad Laboratories) in Tris-glycine buffer at $100 \mathrm{~mA}$ for one hour and at $300 \mathrm{~mA}$ for one hour. The membrane was then washed three times with Tris-buffered saline (TBS) and blocked with 5\% skim milk in TBS for one hour. The membrane was washed three times with TBS containing $0.1 \%$ Tween 20 and three times with TBS, and then equilibrated with $2 \%$ skim milk in TBS for 20 minutes. Immunogenic proteins were detected by incubation of the membrane with human sera $(1: 150)$ overnight at $4^{\circ} \mathrm{C}$. Mouse anti-human IgG conjugated to alkaline phosphatase (Sigma-Aldrich) were added at a dilution of 1:5,000. Before and after addition of the secondary antibody, the membrane was washed three times in TBS containing $0.1 \%$ Tween 20 and three times with TBS. The membrane was incubated with 5-bromo-4-chloro-3-indolyl-phosphate/nitro blue tetrazolium substrate (Sigma-Aldrich) until a desired level of staining was achieved and digitized by a personal densitometer SI (Amersham Biosciences, Piscataway, NJ).

\section{RESULTS}

Glossina sialomes. The profiles of salivary proteins (sialomes) from male and female Glossina, both vector or nonvector species, are shown in Figure 1. Approximately 15-20 bands with molecular weights of 11-240 kD were observed in saliva samples of each Glossina species. Saliva of the three potential vector species of $T$. b. gambiense ( $G$. fuscipes fuscipes, G. tachinoides, and $G$. palpalis gambiensis) had many common bands (approximately 24, 27, 33, 37, 39, 54, 60, 70, $100,110,130$, and $150 \mathrm{kD}$ ) with some variations in intensity of silver staining. Some of these proteins were also detected in G. morsitans morsitans saliva, but non-vector saliva contained more proteins with molecular weights of approximately 50-72 


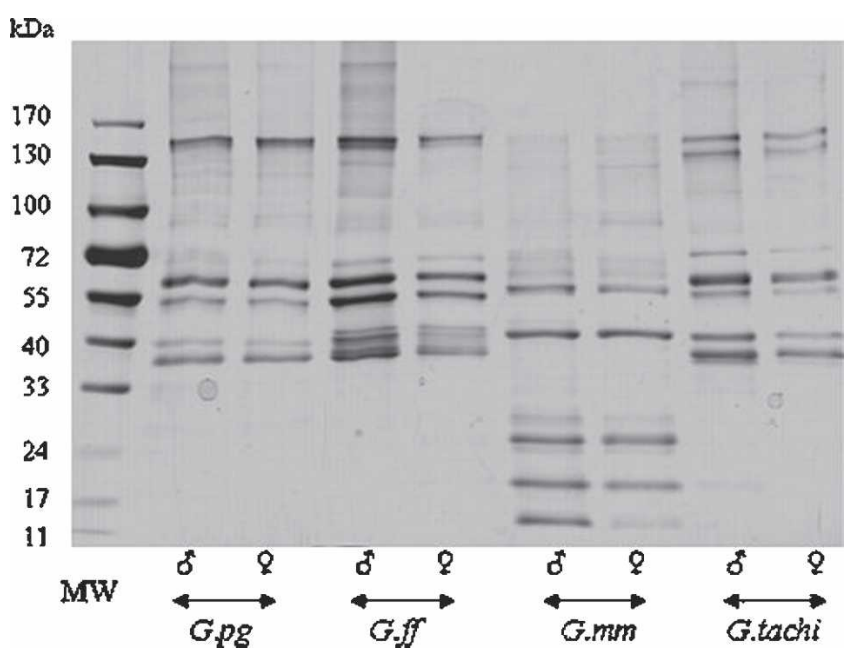

FIGURE 1. Protein composition of saliva from uninfected male (o) and female (q) Glossina species. Shown are three vector species (Glossina palpalis gambiensis [G.pg], G. fuscipes fuscipes [G.ff], G. tachinoides [G.tachi]) and 1 non-vector species (G. morsitans morsitans [G.mm]) of Trypanosoma brucei gambiense. Saliva were analyzed by $4-15 \%$ gradient one-dimensional sodium dodecyl sulfatepolyacrylamide gel electrophoresis under non-reducing conditions and silver staining. Molecular mass markers (MW) are shown on the left in kilodaltons $(\mathrm{kDa})$.

$\mathrm{kD}$ when compared with vector species. Low molecular weight proteins (11-33 kD) were found in the eight saliva samples, but the intensity of silver staining appeared higher in G. morsitans morsitans saliva. The protein profile of Glossina saliva appeared to be species specific, with strong similarities between vector species and important differences in nonvector species G. morsitans morsitans. The composition of saliva appeared similar in females and males for all Glossina species.

Detection of immunogenic proteins according to Glossina species. We evaluated the immunogenic profile of Glossina salivary proteins (immuno-sialome) by immunoblotting with a pool of sera of exposed but non-infected persons (ENI group) (Figure 2). An IgG antibody response specific to saliva samples of each Glossina species was detected and the immunogenic profile appeared to be different according to the Glossina species.

For each saliva sample, 10-15 bands were detected. Some bands $(37,39,54,60,70,95$, and $>170 \mathrm{kD})$ were common to the four species tested. However, the immunogenic profile of non-vector saliva was different from that obtained with saliva of vector species. This difference was closely linked to the immunogenicity of specific proteins observed only in G. morsitans morsitans saliva $(50,55,65$, and $72 \mathrm{kD}$; Figure 2). We also detected a specific band of approximately $42 \mathrm{kD}$ in $G$. fuscipes fuscipes saliva (Figure 2). In addition, the intensity of immunogenic bands differed according to Glossina species. For example, the double band of approximately $130 \mathrm{kD}$ detected in the saliva of the four species (Figure 1) showed higher immunogenic characteristics only in G. morsitans morsitans. In addition, we observed a signal that had a staircase appearance at the low molecular weight range in the vector species. No relevant differences were observed in the immunogenic profile between male and female saliva for any of the Glossina species. Thus, immunogenicity of salivary proteins did not appear to be sex dependent for any Glossina species.

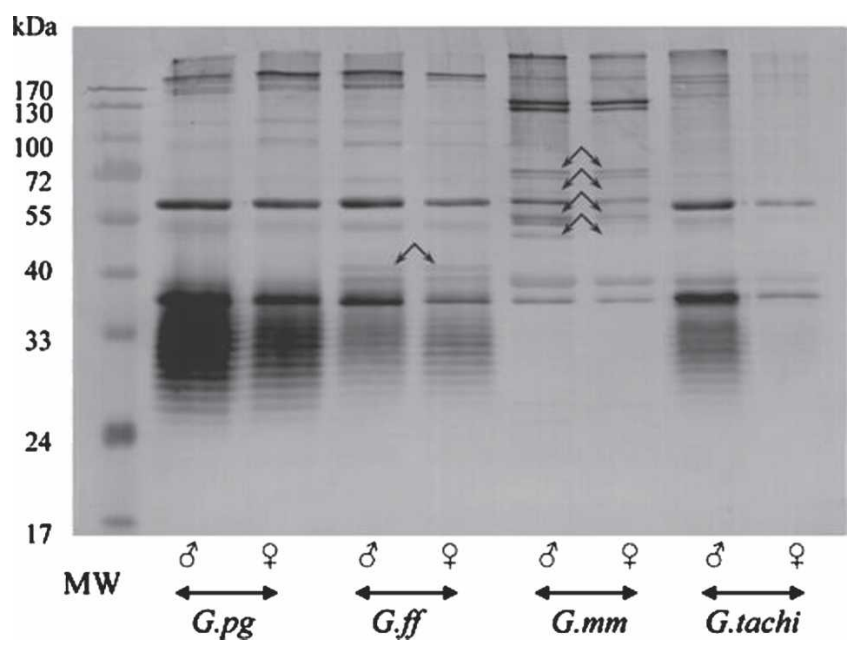

Figure 2. IgG immunogenic profile of salivary proteins in male (ठ) and female (ㅇ) Glossina species in exposed but non-infected persons. Arrows in gel show immunogenic salivary proteins specific to G.ff and G.mm species. For definitions of abbreviations, see Figure 1. Molecular mass markers (MW) are shown on the left in kilodaltons $(\mathrm{kDa})$.

Detection of immunogenic proteins of $G$. fuscipes fuscipes saliva according to the trypanic status of exposed individuals. We evaluated the immunogenicity of salivary proteins during chronic evolution of sleeping sickness by comparing the immune profiles of $G$. fuscipes fuscipes saliva between individual groups (pools of sera) with varying trypanic status: exposed but non-infected persons (ENI group), infected individuals ( $\mathrm{P} 1$ and $\mathrm{P} 2$ groups), and negative controls (CHU group). Results were obtained for saliva from $G$. fuscipes fuscipes because this species is the main vector of $T$. $b$. gambiense in the Democratic Republic of Congo. Figure 3 shows results for male G. fuscipes fuscipes saliva; similar results for each group were observed in females and mixed (male plus female) saliva samples. We observed four major bands (37, 54,60 , and $150 \mathrm{kD}$ ) in the three exposed groups regardless of the HAT status. In addition, four minor bands with low immunogenicity $(90,95,110$, and $125 \mathrm{kD})$ were detected in these three groups. The infected groups ( $\mathrm{P} 1$ and $\mathrm{P} 2)$ showed similar immunogenic profiles. In contrast, a difference in the immunogenic profiles was detected between infected patients (P1 and P2 groups) and exposed but non-infected individuals (ENI group). Some salivary proteins (four bands between 37 $\mathrm{kD}$ and $42 \mathrm{kD}$ ) were immunogenic only in the ENI group. Surprisingly, weak intensity of three immunogenic proteins (54, 60, and $150 \mathrm{kD})$, the major 37-kD band, and the previously observed staircase signal were observed in the $\mathrm{CHU}$ group.

Analysis of the individual immunogenic profile in $G$. fuscipes fuscipes saliva. We investigated the immunogenic profile of exposed but non-infected (ENI) and infected (P1) individuals to mixed $G$. fuscipes fuscipes saliva (female plus male). This study was carried out on nine ENI patients (Figure 4A) who were randomly selected, and on all persons in the P1 group ( $\mathrm{n}=7$; Figure $4 \mathrm{~B})$.

The profiles of individuals in the ENI group were heterogeneous, which suggested variability in the immunogenicity of salivary proteins among individuals with the same trypanic status. The four major immunogenic bands $(37,54,60$, and 


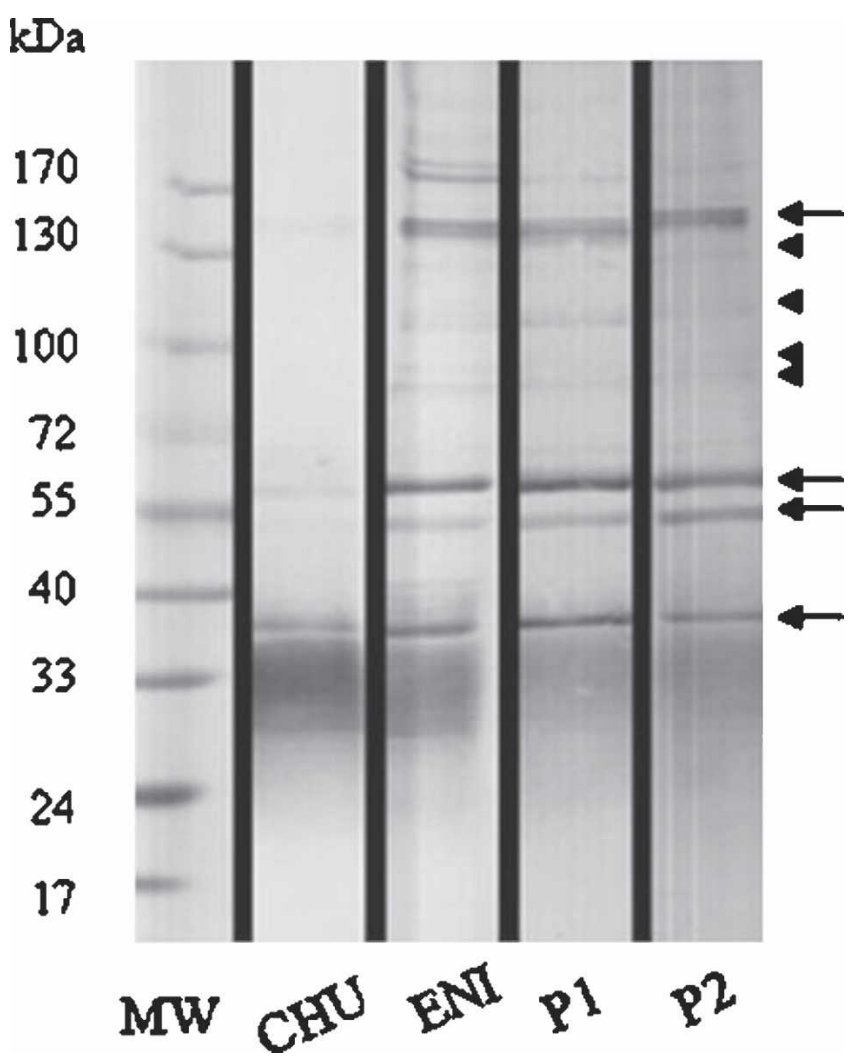

FIGURE 3. IgG immunogenic profile of Glossina fuscipes fuscipes saliva in three patients groups (ENI, P1, and P2) and to the nonexposed group (CHU). Arrows shows four major bands and arrowheads show four minor bands commonly detected in the three exposed groups. ENI = exposed but non-infected individuals with no positive results for four tests; $\mathrm{P} 1$ = exposed and infected patients in the first stage of the disease who had positive results for four tests; P2 = exposed and infected patients in the second stage of the disease who had positive results for four tests. Molecular mass markers (MW) are shown on the left in kilodaltons $(\mathrm{kDa})$.

$150 \mathrm{kD}$ ) and two minor bands with low immunogenicity (90 and $110 \mathrm{kD}$ ) differed in intensity among persons in the ENI group. In addition, such individual variations were also observed for ENI-specific proteins of approximately 37-42 kD.

In contrast, a similar profile of immunogenic salivary proteins was observed among persons in the $\mathrm{P} 1$ group. Most individuals in this group showed a specific IgG antibody response to the four major bands $(37,54,60$, and $150 \mathrm{kD})$ with particularly high immunogenicity for three of them $(37,60$, and $150 \mathrm{kD})$. In addition, this homogeneity of the profile of P1 individuals was observed with two bands having low immunogenicity (70 and $110 \mathrm{kD}$ ). Most individual profiles (ENI and P1) had the previously observed staircase signal at low molecular weights.

\section{DISCUSSION}

In the present study, specific immune responses to salivary proteins of Glossina were detected in humans residing in an area endemic for a sleeping sickness. We demonstrated that the specific antibody response to these immunogenic proteins was dependent on the Glossina species and the infection status of studied individuals.

Previous studies on Glossina saliva had been carried out
A

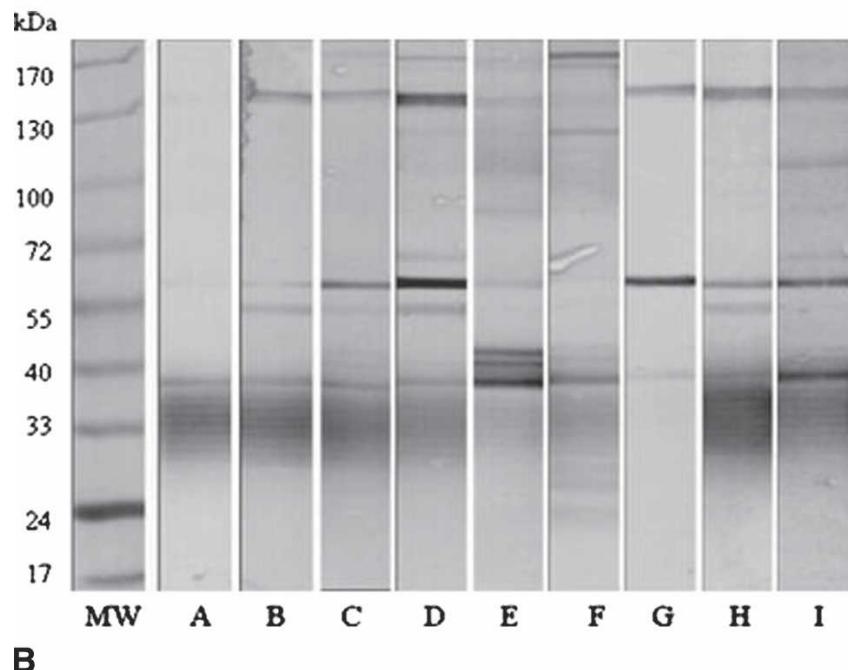

B

$\mathrm{kDa}$

170
130

100

72

55

40

33

24
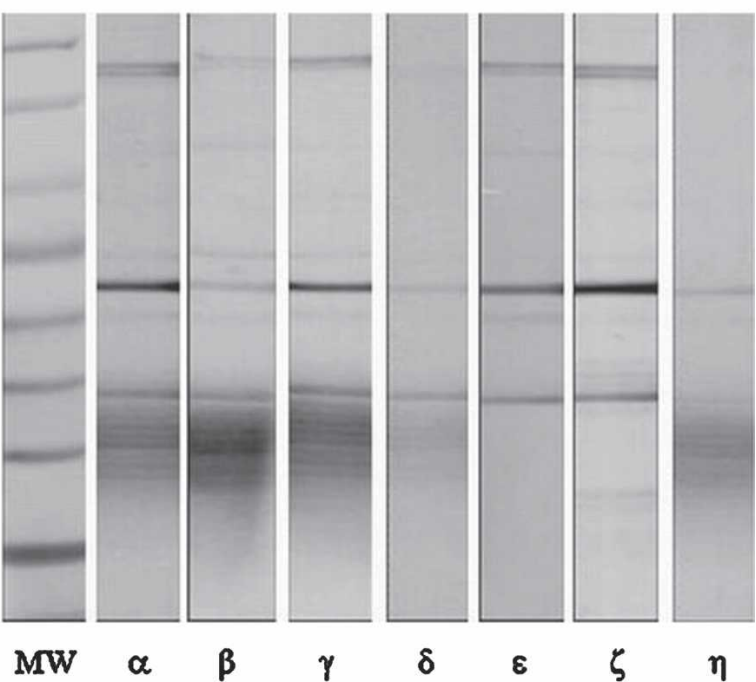

Figure 4. A, Individual IgG immunogenic profile of Glossina fuscipes fuscipes saliva in nine individuals (A-I) from the exposed but non-infected group (ENI). B, Individual IgG immunogenic profile of $G$. fuscipes fuscipes saliva in seven individuals $(\alpha-\eta)$ from the exposed and infected group (P1). Molecular mass markers (MW) are shown on the left in kilodaltons $(\mathrm{kDa})$.

principally on salivary glands (cDNA bank or protein extraction) of non-vector Glossina species of T. b. gambiense ( $G$. morsitans morsitans and $G$. morsitans centralis), but salivary glands contain structural proteins that are not secreted during biting. ${ }^{19}$ Nevertheless, they enable identification of some proteins with pharmacologic properties involved in inhibition of the human host hemostasis response. ${ }^{26}$ In the present study, the salivation technique used enabled analysis of biologic material similar to saliva injected in the vertebrate host during natural blood feeding. This enabled identification of components involved in the host/vector relationship. The sequencing of an extensive set of expressed sequence tags of the G. morsitans morsitans salivary gland is currently being carried out and should confirm the effectiveness of our salivation technique. $^{27}$

In the present study, analysis of sialomes identified 15-20 bands by one-dimensional SDS-PAGE. It would be useful to 
determine whether one band corresponds to one or more proteins. Such identification could be performed by separating the salivary proteins by two-dimensional electrophoresis and analyzing them by mass spectrometry. However, we have shown that numerous bands are common to Glossina species, but other bands are present only in potential vector species (G. fuscipes fuscipes, G. tachinoides, and G. palpalis gambiensis) and non-vector species (G. morsitans morsitans) of T. $b$. gambiense. This difference in saliva composition, including the presence or absence of certain salivary proteins, could be involved in vector capacity of the Glossina species. For example, a salivary protein may be essential for continuation of the parasite maturation cycle in the vector, as suggested in the Anopheles mosquito. ${ }^{28}$

To our knowledge, the present study is also the first description of Glossina immuno-sialome carried out by investigating the specific human IgG response to Glossina saliva in a population exposed to HAT. Previously, hypersensitivity reactions of an immediate or delayed type were observed in humans, but specific salivary components involved had not been identified. ${ }^{18,20}$ Moreover, a previous study described sialome and immunogenic salivary proteins of $G$. morsitans centralis that were detected by $\operatorname{IgG}$ antibodies from sensitized rabbits. ${ }^{19}$

Comparison of immuno-sialomes of the four Glossina species shows a difference in the profile of immunogenic proteins according to species. This difference was greater between Glossina vector species or non-vector species of T. b. gambiense, as we observed in sialome analyses. Most of these immunogenic proteins were common to the four Glossina species. Nevertheless, the immunoblotting approach distinguished immunogenic proteins specific to Glossina species (42 $\mathrm{kD}$ specific for G. fuscipes fuscipes and 50, 55, 65, and $72 \mathrm{kD}$ specific for G. morsitans morsitans). Species-specific antigens were also identified in Phlebotomus and Aedes species. ${ }^{29,30}$ The difference in immunogenic protein composition in Glossina species may also be involved in transmission of the parasite. For example, the immune response induced by specific proteins of non-vector $G$. morsitans morsitans (50, 55, 65, and $72 \mathrm{kD}$ ) could play a role in preventing infection permissiveness. It has been demonstrated that the specific immune response to one Phlebotomus salivary antigen (PpSP15) can confer protection against the development of leishmaniasis in animal models. ${ }^{31}$

Another difference between vector and non-vector Glossina species was also observed. A signal $(<37 \mathrm{kD})$ in the form of bands in a staircase pattern was only detected in saliva of Glossina vector species (G. fuscipes fuscipes, G. tachinoides, and $G$. palpalis gambiensis). One hypothesis is that this signal could be an artifact of handling. In addition, endosymbiont Sodalis glossinidus bacteria can be found at the level of salivary glands of certain Glossina species, but are absent in $G$. morsitans morsitans salivary glands. ${ }^{32}$ Another hypothesis is that proteins of these bacteria are secreted during Glossina bites and induce a specific immune response in humans. The staircase signal was also detected in $G$. fuscipes fuscipes saliva using sera from the CHU group, which was not exposed to Glossina bites and thus was not exposed to $S$. glossinidus. This surprising result may also represent the same artifact of handling or detection of cross-reactivity with compounds of other bacteria. Moreover, numerous bacteria express proteins on their surface that bind to the Fc regions of $\mathrm{IgG}$, which will enable these bacteria to be covered with host protein and escape the defense mechanism of the host. ${ }^{33}$ Some bands observed in the presented immunoblottings might be linked to this bacterial capacity. Such proteins from bacterial surfaces could be present in Glossina saliva and thus bind IgG antibodies (human antibodies in serum and/or secondary antibodies used in the assay) in a non-specific manner. In addition, three bands with low immunogenicity $(54,60$, and $150 \mathrm{kD})$ were detected with the sera of the CHU group, which also suggests immune cross-reactivity. This could be due to crossreactions between common epitopes of arthropod salivary proteins from mosquitoes, horseflies, and sand flies. Further investigations are necessary to define the immunologic specificity of salivary proteins of Glossina.

We also studied the relationship of immuno-sialome to the specific immunogenic profile relative to the trypanic status of individuals (exposed but non-infected [ENI] and infected [P1 and $\mathrm{P} 2]$ ). We investigated the specific immune response to $G$. fuscipes fuscipes because this species is the main vector species of T. b. gambiense in the Democratic Republic of Congo. Immunoblotting with a pool of sera from these three groups resulted in detection of immunogenic proteins that were classified into two clusters according to their intensity of immunogenicity: the four major bands with high immunogenicity $(37,54,60$, and $150 \mathrm{kD})$ and four minor bands with low immunogenicity $(90,95,110$, and $125 \mathrm{kD})$. We observed a similar immunologic profile for these two clusters in the three exposed groups. This resulted in detection of common salivary proteins that are immunogenic in all groups exposed to G. fuscipes fuscipes bites regardless of the infection status of the patients. However, some variations in detection of immunogenic proteins were observed between the ENI and P1/P2 groups. The $\mathrm{IgG}$ response to smaller salivary proteins (four bands between $37 \mathrm{kD}$ and $42 \mathrm{kD}$ ) was detected only in the ENI group. This suggests that the immunogenicity of some salivary proteins decreases with the infection status of sleeping sickness (exposed versus infected), which could be due to immunosuppression observed during development of sleeping sickness. ${ }^{34}$

Analysis of the immunologic profile of exposed (ENI group) and infected (P1 group) individuals also confirmed group-dependent differences according to the exposed versus infected status. The ENI group showed heterogeneity in immunogenic profiles between individuals, which suggested that exposed but non-infected individuals have a different immune response to Glossina salivary proteins. In contrast, the immunogenic profile was similar among infected P1 individuals for whom three major bands $(37,60$, and $150 \mathrm{kD})$ with high immunogenicity were commonly detected.

Since the mid 1990s, several studies have investigated the immune response of mammalian hosts to arthropod bites. ${ }^{35}$ Qualitative and quantitative evaluation of specific human antibody responses to arthropod salivary proteins may be a useful marker of exposure to vector-borne diseases, similar to markers for malaria in Senegal and for Chagas disease and visceral leishmaniasis in Brazil. ${ }^{12,16,17}$

Selection of immunogenic proteins for elaboration of an immune marker of exposure to Glossina requires knowledge of vector saliva composition in proteins (sialome) and immunogenic characteristics of these proteins (immuno-sialome). Candidate proteins must be expressed in saliva of most Glossina species, or at least be common to vector Glossina species. 
In several hematophagous species, the male does not take a blood meal, which is reflected in salivary morphology and secretions. ${ }^{36}$ Both female and male Glossina could be vectors of Trypanosoma, and we have shown that both sexes have similar immunogenic profiles of salivary proteins. Since the observed immune response must be specific to Glossina salivary antigens, the protein containing potential common epitopes with other vector arthropods would have to be eliminated from the pool of candidate proteins. Furthermore, immunogenic proteins specific to a Glossina species $(42 \mathrm{kD}$ for G. fuscipes fuscipes and 50,55, 65, and $72 \mathrm{kD}$ for $G$. morsitans morsitans in our study) should also be markers of distinguishing specific exposure to the bite of one Glossina species.

Candidate proteins should have immunogenic properties enabling detection of specific antibodies in all persons exposed to Glossina bites, whether or not the persons are infected. Several immunogenic proteins of interest were emphasized in our results. Major proteins were identified in ENI and P1 individuals, including 37-, 60-, and 150-kD bands. However, these bands, except for the $37-\mathrm{kD}$ band, also appeared to be immunogenic in the unexposed $\mathrm{CHU}$ group but their immunogenicity was low. Among the minor proteins, only the $110-\mathrm{kD}$ protein was immunogenic in most individuals exposed to Glossina bites, but it showed weak immunogenicity in our study. We have not identified a marker protein, and our results represent a first attempt to characterize the immunogenic profile of Glossina salivary proteins in an exposed population. Nevertheless, this study indicates the feasibility of elaboration of an immuno-epidemiologic marker of exposure based on antibody response to salivary proteins.

Further studies on other sleeping sickness transmission foci are needed to confirm these results. The immuno-proteomic approach using two-dimensional electrophoresis could identify immunogenic proteins specific for exposure to Glossina bites and could be used for mass spectrometric characterization of proteins. After immunogenic proteins are identified, selected proteins could be produced in their recombinant form and a quantitative method (enzyme-linked immunosorbent assay) could be used to evaluate specific individual antibody titers to these proteins in the framework of large-scale immuno-epidemiologic studies. These immunologic results could be compared with exposure (as evaluated by an entomologic approach) and morbidity data.

The development of an immuno-epidemiologic marker of exposure to Glossina bites could represent a tool complementary to those currently available, which include diagnostic tests enabling evaluation of morbidity and environmental data (satellite data, climate models, entomologic information, and cartography) that define where and when populations have been exposed to vectors and parasites. ${ }^{37}$ Such a marker could be used to estimate the exposure of individuals to Glossina bites and be an indicator of risks of HAT transmission.

Received May 31, 2006. Accepted for publication July 31, 2006.

Acknowledgments: We gratefully acknowledge the population of the Bandundu area for participation in the study. We thank G. Cuny for access to tsetse flies bred in an insectarium, A. M, Dupuy and the occupational health team of Lapeyronie Hospital (Montpellier, France) for access to negative sera from personnel who had never been to Africa; and J. P. Brizard for access to the densitometer.

Financial support: The study in the Democratic Republic of Congo was supported by the Institut de Médecine et Epidémiologie Afri- caine and the Institut de Recherche pour le Développement. Sylvie Cornelie holds a fellowship from the French Fonds Inkerman and the Fondation Singer Polignac, David Courtin holds a fellowship from the French Fondation des Treilles.

Authors' addresses: Anne Poinsignon, Sylvie Cornelie, and Francois Simondon, Epidémiologie and Prévention, Unité de Recherche 024, Institut de Recherche pour le Développement, 911 Avenue Agropolis, BP 64501, 34394 Montpellier CEDEX 5, France, Telephone: 334-67-41-63-32, Fax: 33-4-67-41-63-30, E-mails: anne.poinsignon@ mpl.ird.fr, cornelie@mpl.ird.fr, and francois.simondon@mpl.ird.fr. Franck Remoue, Epidémiologie and Prévention, Unité de Recherche 024, Institut de Recherche pour le Développement Dakar, Routes des Pères Maristes, BP 1386, 18524 Dakar, Senegal, Telephone 221849-3555, Fax: 221-832-4307, E-mail: remoue@ird.sn. Pascal Grébaut, Laboratoire de Recherche et de Coordination contre les Trypanosomoses, Centre de Coopération International de Recherche Agronomique pour le Développement, Institut de Recherche pour le Développement, Unité Mixte de Recherche 177, Interactions HôtesVecteurs-Parasites dans les Trypanosomoses, TA 207 G, Campus International de Baillarguet, 34398 Montpellier CEDEX 5, France, Telephone and Fax: 33-4-67-59-39-25, E-mail: pascal.grebaut@ mpl.ird.fr. David Courtin, Unité de Recherche 010, Institut de Recherche pour le Développement, Santé de la Mère et de L'Enfant en Milieu Tropical, Faculté de Pharmacie, 4 Avenue de L'Observatoire, 75270 Paris, France, Telephone: 33-1-53-73-96-17, Fax: 33-1-53-73-9617, E-mail: d.courtin@gmail.com. Andre Garcia, Institut de Recherche pour le Développement Benin, La Résidence Les Cocotiers 08, BP 841 Cotonou, Benin, Telephone: 229-21-30-98-21, Fax: 229-21-9545-51-14, E-mail: andre.garcia@ird.fr.

\section{REFERENCES}

1. Boa YF, Traore MA, Doua F, Kouassi-Traore MT, Kouassi BE, Giordano C, 1988. The different present-day clinical picture of human African trypanosomiasis caused by T. b. gambiense. Analysis of 300 cases from a focus in Daloa, Ivory Coast. Bull Soc Pathol Exot Filiales 81: 427-444.

2. World Health Organization, 1998. Control and surveillance of African trypanosomiasis. Report of a WHO expert committee. World Health Organ Tech Rep Ser 881: 1-114.

3. van Nieuwenhove S, Betu-Ku-Mesu VK, Diabakana PM, Declercq J, Bilenge CM, 2001. Sleeping sickness resurgence in the DRC: the past decade. Trop Med Int Health 6: 335-341.

4. World Health Organization, 2006. Human African trypanosomiasis. Wkly Epidemiol Rec 8: 69-80.

5. Hervouet JP, Laveissière C, 1987. Facteurs humains dans la transmission de la maladie du sommeil. Med Trop Nella Cooperazione Allo Sviluppo 3: 72-78.

6. Laveissiere C, Sane B, Meda HA, 1994. Measurement of risk in endemic areas of human African trypanosomiasis in Côte d'Ivoire. Trans R Soc Trop Med Hyg 88: 645-648.

7. Kuzoe F, Schofield C, 2005. Strategic Review of Traps and Targets for Tsetse and African Trypanosomiasis Control. Geneva: World Health Organization. TDR/IDE/TRY/05.1.

8. Ribeiro JM, Francischetti IM, 2003. Role of arthropod saliva in blood feeding: sialome and post-sialome perspectives. Annu Rev Entomol 48: 73-88.

9. Remoue F, Cornelie S, NGom A, Boulanger D, Simondon F, 2005. Immune responses to arthropod bites during vectorborne diseases. Garraud $\mathrm{O}$, ed. Update in Tropical Immunology. Herala, India: Transworld Research Network, 377-400.

10. Schwartz BS, Ribeiro JM, Goldstein MD, 1990. Anti-tick antibodies: an epidemiologic tool in Lyme disease research. Am J Epidemiol 132: 58-66.

11. Lane RS, Moss RB, Hsu YP, Wei T, Mesirow ML, Kuo MM, 1999. Anti-arthropod saliva antibodies among residents of a community at high risk for Lyme disease in California. Am J Trop Med Hyg 61: 850-859.

12. Nascimento RJ, Santana JM, Lozzi SP, Araujo CN, Teixeira AR, 2001. Human IgG1 and IgG4: the main antibodies against Triatoma infestans (Hemiptera: Reduviidae) salivary gland proteins. Am J Trop Med Hyg 65: 219-226.

13. Palosuo K, Brummer-Korvenkontio H, Mikkola J, Sahi T, Reunala $\mathrm{T}, 1997$. Seasonal increase in human $\mathrm{IgE}$ and $\mathrm{IgG} 4$ anti- 
saliva antibodies to Aedes mosquito bites. Int Arch Allergy Immunol 114: 367-372.

14. Reunala T, Brummer-Korvenkontio H, Palosuo K, Miyanij M, Ruiz-Maldonado R, Love A, Francois G, Palosuo T, 1994. Frequent occurrence of $\mathrm{IgE}$ and $\mathrm{IgG} 4$ antibodies against saliva of Aedes communis and Aedes aegypti mosquitoes in children. Int Arch Allergy Immunol 104: 366-371.

15. Konishi E, 1990. Distribution of immunoglobulin G and E antibody levels to salivary gland extracts of Aedes albopictus (Diptera: Culicidae) in several age groups of a Japanese population. J Med Entomol 27: 519-522.

16. Barral A, Honda E, Caldas A, Costa J, Vinhas V, Rowton ED, Valenzuela JG, Charlab R, Barral-Netto M, Ribeiro JM, 2000. Human immune response to sand fly salivary gland antigens: a useful epidemiological marker? Am J Trop Med Hyg 62: 740745 .

17. Remoue F, Cisse B, Ba F, Sokhna C, Herve J-P, Boulanger D, Simondon F, 2006. Evaluation of the antibody response to Anopheles salivary antigens as a potential marker of risk of malaria. Trans $R$ Soc Trop Med Hyg 100: 363-370.

18. Gordon R, Crewe W, 1948. The mechanisms by witch mosquitoes and tsetse flies obtain their blood meals, the histology of the lesions produced and the subsequent reactions of the mammalian host; together with some observations on the feeding of Chrysops and Cimex. Ann Trop Med Parasitol 42: 334356.

19. Ellis JA, Shapiro SZ, ole Moi-Yoi O, Moloo SK, 1986. Lesions and saliva-specific antibody responses in rabbits with immediate and delayed hypersensitivity reactions to the bites of Glossina morsitans centralis. Vet Pathol 23: 661-667.

20. Penchenier L, Terrasse C, Diallo BP, Eouzan JP, 1986. Le Contact Homme-Glossine: Réactions Immunitaires à la Salive de Glossine. Communication OCCGE no. 27/IRTO/RAP/84. Bouake, Côte d'Ivoire: Institut de Recherches sur la Trypanosomiase et l'Onchocercose.

21. Courtin D, Jamonneau V, Mathieu J-F, Koffi M, Milet J, Yeminanga CS, Kumeso VK, Cuny G, Bilengue CM, Garcia A, 2006. Comparison of cytokine plasma levels in human African trypanosomiasis. Trop Med Int Health 11: 647-653.

22. Magnus E, Vervoort T, van Meirvenne N, 1978. A cardagglutination test with stained trypanosomes (C.A.T.T.) for the serological diagnosis of $T$. B. gambiense trypanosomiasis. Ann Soc Belg Med Trop 58: 169-176.

23. van Meirvenne N, Magnus E, Buscher P, 1995. Evaluation of variant specific trypanolysis tests for serodiagnosis of human infections with Trypanosoma brucei gambiense. Acta Trop 60: 189-199.
24. Gidudu AM, Cuisance D, Reifenberg JM, Frezil JL, 1995. Improving the salivation technique in the tsetse fly for the detection of infective metatripanosomes: study of the effect of biologic and non-biologic factors in the probing behavior of the tsetse fly. Rev Elev Med Vet Pays Trop 48: 153-160.

25. Shevchenko A, Wilm M, Vorm O, Mann M, 1996. Mass spectrometric sequencing of proteins silver-stained polyacrylamide gels. Anal Chem 68: 850-858.

26. Cappello M, Bergum PW, Vlasuk GP, Furmidge BA, Pritchard DI, Aksoy S, 1996. Isolation and characterization of the tsetse thrombin inhibitor: a potent antithrombotic peptide from the saliva of Glossina morsitans morsitans. Am J Trop Med Hyg 54: 475-480.

27. Aksoy S, Berriman M, Hall N, Hattori M, Hide W, Lehane MJ, 2005. A case for a Glossina genome project. Trends Parasitol 21: $107-111$.

28. Hirai M, Wang J, Yoshida S, Ishii A, Matsuoka H, 2001. Characterization and identification of exflagellation-inducing factor in the salivary gland of Anopheles stephensi (Diptera: Culicidae). Biochem Biophys Res Commun 287: 859-864.

29. Volf P, Rohousova I, 2001. Species-specific antigens in salivary glands of phlebotomine sandflies. Parasitology 122: 37-41.

30. Brummer-Korvenkontio H, Palosuo T, Francois G, Reunala T, 1997. Characterization of Aedes communis, Aedes aegypti and Anopheles stephensi mosquito saliva antigens by immunoblotting. Int Arch Allergy Immunol 112: 169-174.

31. Valenzuela JG, Belkaid Y, Garfield MK, Mendez S, Kamhawi S, Rowton ED, Sacks DL, Ribeiro JM, 2001. Toward a defined anti-Leishmania vaccine targeting vector antigens: characterization of a protective salivary protein. J Exp Med 194: 331342.

32. Cheng Q, Aksoy S, 1999. Tissue tropism, transmission and expression of foreign genes in vivo in midgut symbionts of tsetse flies. Insect Mol Biol 8: 125-132.

33. Boyle MDP, 1990. Bacterial Immunoglobulin Binding Proteins. San Diego CA, ed. San Diego: Academic Press.

34. Vincendeau P, Okomo-Assoumou MC, Semballa S, Fouquet C, Daulouede S, 1996. Immunology and immunopathology of African trypanosomiasis. Med Trop 56: 73-78.

35. Schoeler GB, Wikel SK, 2001. Modulation of host immunity by haematophagous arthropods. Ann Trop Med Parasitol 95: 755771.

36. James AA, 1994. Molecular and biochemical analysis of the salivary glands of vector mosquitoes. Bull Inst Pasteur 92: 133-150.

37. Thomson MC, Connor SJ, 2000. Environmental information systems for the control of arthropod vectors of disease. Med Vet Entomol 14: 227-244. 